\title{
ANALYTIC MODELING OF LOSS AND CROSS-COUPLING IN CAPACITIVE MICROMACHINED ULTRASONIC TRANSDUCERS
}

\author{
Ayhan Bozkurt, $\dagger$ F. L. Degertekin, Abdullah Atalar, $† B$. T. Khuri-Yakub \\ Department of Electrical and Electronics Engineering: Bilkent University Bilkent 06533 Ankara, Turkey. \\ †Edward L.Ginzton Laboratory, Department of Electrical Engineering, Stanford University, Stanford, CA 94305.
}

\begin{abstract}
The structural loss mechanism of capacitive micromacined ultrasonic transducer (cMUT) is investigated using finite element analysis and the normal mode theory. A single micromachined tranducer membrane on an infinite silicon substrate is simulated by incorporating absorbing boundary conditions in the finite element method. This enables direct evaluation of the mechanical impedance of the membrane. Furthermore, the field distribution along the thickness of the silicon substrate due to outward radiating wave modes is obtained. The normal mode theory is applied to extract the contributions of different wave modes to the complicated field distributions. It is found that, the lowest order Lamb wave modes are responsible for the loss. Evaluation of absolute and relative power losses due to individual modes indicate that the lowest order anti-symmetric $\left(A_{0}\right)$ mode is the dominant radial mode in agreement with experimental measurements. The results of the analysis are used to derive a detailed equivalent circuit model of a cMUT with structural loss.
\end{abstract}

\section{INTRODUCTION}

Air-coupled and immersion capacitive ultrasonic transducers have long existed and their characteristics and performance have been exhaustively evaluated. Recent developments in micromachining techniques have led to the design of microfabricated versions of the devices [1]. The cMUT is constructed on a silicon wafer using a micromachining process and consists of cells that have dimensions in the order of tens of microns [2]. As a transducer of reasonable size has a size expressed in millimeters, thousands of this devices need to be placed on the same substrate [3]. Consequently, an individual transducer cell, instead of being clamped at its ends, resides on a large substrate and couples power to radially outward wave modes. Furthermore, this power is received by the neighboring transducer elements creating crosscoupling between individual cells of the transducer. Although some theory explaining the behavior of a single cell has been proposed, the above mentioned power loss and cross-coupling effects still remain unexplained. As main motivational forces behind trans- ducer development include applications in air-coupled nondestructive evaluation (NDE), analysis methods that include the loss mechanisms in cMUTs need to be developed for the design of efficient transducers for ultrasonic transmission into air. Likewise, designs for 3-D immersion imaging using 2-D transducer matrices require a good understanding of coupling between array elements. All of these call for the development of models close to reality. The starting point for the analytical derivations is the theory developed for discrete elements and these derivations will include the inherent physical properties of the new transducer technology. In this paper, we present an analytic model of the cMUT which includes effects of substrate loss. The model is developed by the help of numerical tools (finite element method simulations) and analysis of wave modes in the substrate by the normal mode theory.

\section{Finite Element MODEling}

The cMUT under analysis is considered to have a structure as depicted in Fig. 1. The structure is an

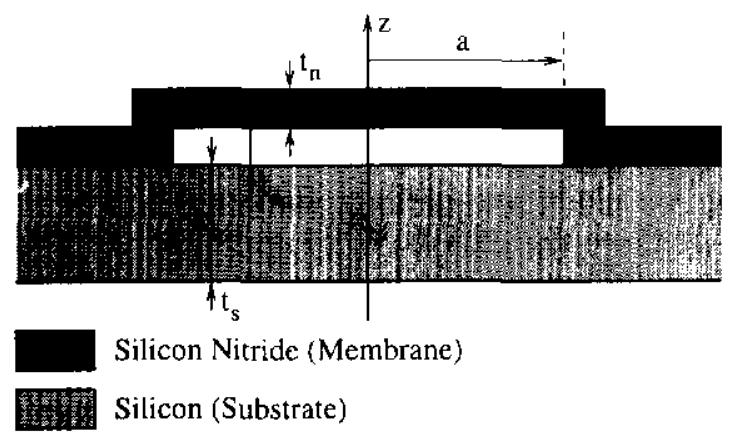

Fig. 1. Cross-sectional view of cMUT structure.

object of rotation about the z-axis with a being the radius of the active membrane area. The membrane consists of a silicon nitride layer of thickness $t_{n}$ and the membrane substrate separation is $t_{a}$. The driving force for the membrane's vibration is the electrostatic attraction force between a metal electrode deposited on the membrane and the silicon substrate. The following device dimensions have been used throughout the analyses (Table I): The listed dimensions results 
TABLE I

DEVICE DIMENSIONS FOR THE ANALYSES.

\begin{tabular}{l|c|r} 
Parameter & Symbol & Value \\
\hline Membrane thickness & $t_{n}$ & $1.0 \mu \mathrm{m}$ \\
Airgap thickness & $t_{a}$ & $1.0 \mu \mathrm{m}$ \\
Substrate thickness & $t_{s}$ & $500.0 \mu \mathrm{m}$ \\
Membrane Radius & $a$ & $45.0 \mu \mathrm{m}$
\end{tabular}

in a device whose first order resonance is at $2.3 \mathrm{MHz}$.

As we are primarily concerned with the power that the membrane couples to the radially outward propagating wave modes of the substrate, we need to have an absorbing boundary at the radial edges of our model. Levander [4] and Cerjan et al. [5] have used lossy material boundaries to absorb waves incident on model boundaries. Later, Berenger's [6] Perfectly Matched Layer (PML) for electromagnetic waves was shown to be applicable to elastic wave propagation problems by Chew et al. [7] and was used in a number of applications [8], [9], [10]. In this study, we use a lossy medium of considerable length to absorb the outward propagating waves. Fig. 2 depicts the finite element model involved in the analysis. The attenua-

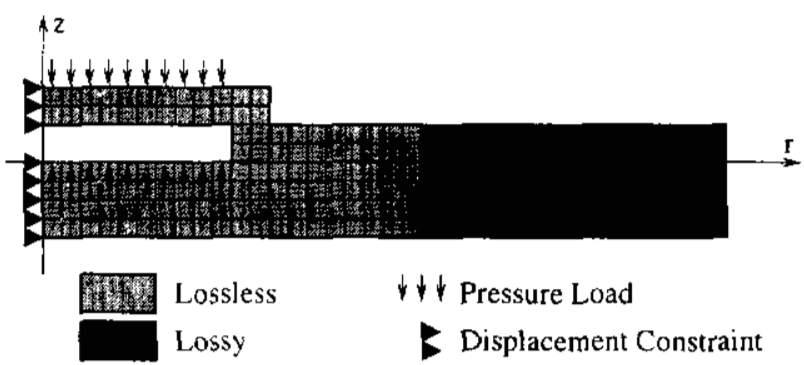

Fig. 2. Finite-element model of the cMUT.

tion in the lossy part of the model has to be kept small to minimize the impedance mismatch at the boundary of the lossless and lossy regions. On the other hand, the lossy region should provide enough attenuation so that incident waves at the model boundary should be well attenuated and the reflected waves at that end should not result in a significant standing wave pattern. This can be achieved by having a very long attenuating region as compared to the largest wavelength.

The structure of Fig. 2 is used to run harmonic analyses using ANSYS $5.4^{1}$ to test the validity of our method. The indicated pressure loads are applied together with the displacement constraints. ANSYS

${ }^{1}$ (C) SAS IP, Inc. enables the use of an axisymmetric model, therefore we were able to model the cMUT as the 2-D structure of Fig. 2. As a first check, we look at the particle displacement at the surface of the substrate as a function of radial distance. Displacement is expected to decay monotonically with $\sqrt{r}$ [11]. Fig. 3 shows a plot of nodal displacement magnitude as a function of radial distance at an excitation frequency of $1.5 \mathrm{MHz}$. The radial distances up to $40 \mathrm{~mm}$ are

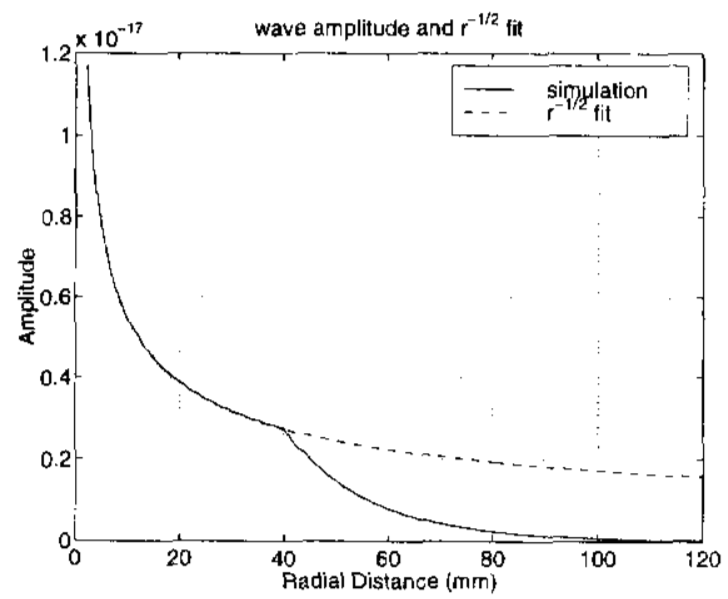

Fig. 3. Magnitude of nodal displacement on substrate.

in the lossless region of the model and, as expected, there is a good agreement between the magnitude and the $1 / \sqrt{r}$ curve shown with the dashed line. For $40 \mathrm{~mm}<r<120 \mathrm{~mm}$, wave magnitude dies away and, as the plot has reasonable smoothness, there is no significant reflection at either boundary.

\section{Normal Mode Analysis}

The finite element analysis also provides the distribution of particle displacement and stress components along the thickness of the silicon substrate generated by the vibrating membrane. One can use these field variables to calculate the total radiation loss to the substrate. However, a deeper understanding can be gained by identifying the different wave modes using the 2-D normal mode decomposition [12]. Since the excitation and propagation of the normal modes are well known, the loss mechanisms can be revealed and can be used to reduce the loss by changing membrane parameters. An arbitrary distribution of particle velocity $\mathbf{v}(x, z)$ and associated stress field $\mathbf{T}(x, z)$ along the thickness of the plate can be written as

$$
\mathbf{v}(x, z)=\sum_{n=0}^{\infty} a_{n}(x) \mathbf{v}_{n}(z)
$$




$$
\mathbf{T}(x, z)=\sum_{n=0}^{\infty} a_{n}(x) \mathbf{T}_{n}(z) \cdot \mathbf{x}
$$

where the orthogonal field distributions $\mathbf{v}_{n}(z)$ and $\mathbf{T}_{n}(z) \cdot \mathbf{x}$ are in fact the field distributions of the propagating Lamb waves in the free silicon plate. Given the frequency range of interest $(1-3.5 \mathrm{MHz})$ and a $0.5 \mathrm{~mm}$ thick silicon plate, the only propagating modes are the two lowest order antisymmetric $\left(A_{0}\right)$ and symmetric $\left(S_{0}\right)$ Lamb wave modes. The mode amplitude $a_{n}$ can be found by substituting the corresponding orthogonal mode expansions for the arbitrary fields in Eq. 1 and 2 in the orthogonality relation

$$
a_{n}=\frac{1}{4 \mathrm{P}_{n n}} \int_{-t_{s}}^{0}\left(-\mathbf{v}_{n}^{*}(z) \cdot \mathbf{T}(z)-\mathbf{v}(z) \cdot \mathbf{T}_{n}^{*}(z)\right) \cdot \mathbf{x} d z
$$

$\mathrm{P}_{n n}$ is the average power flow of the mode per unit width in the $x$-direction for $a_{n}=1$. To apply this methodology to the circularly symmetric geometry, it is inherently assumed that the calculations are performed at a radius which is sufficiently far away from the membrane.

The total power radiated, hence dissipated by the lossy region, $P_{\text {tot }}$ can be found by

$$
P_{t o t}=2 \pi r_{1} \frac{1}{2} \operatorname{Re}\left\{\int_{-t_{s}}^{0}\left(v_{x}^{*} T_{x x}+v_{z}^{*} T_{x z}\right) d z\right\}
$$

where all stress and velocity values are at a cross section at $r=r_{1}$, with $r_{1}$ being inside the lossless part of the model and close to the boundary of the lossy and lossless regions. The radiated power levels of the Lamb wave modes are then found by substituting the results of the finite element analysis in Eq. 3 and using the relation $P_{n}=2 \pi r_{1}\left|a_{n}\right|^{2} \mathrm{P}_{n n}$.

The modal decomposition results show that the $A_{0}$ mode Lamb wave is the dominant source of the radiation loss, especialy at lower frequencies. This can be clearly seen in Fig. 4 where the modal particle velocity field distribution of the $A_{0}$ mode and the result of the FEA calculation at $1 \mathrm{MHz}$ are plotted. The antisymmetric nature of the radiated field is very similar to the $A_{0}$ mode. Radiated power calculation results support this conclusion. The total radiated power loss as a function of frequency is plotted in Fig. 5 as the solid line. As expected, the radiated power is maximum at the resonance frequency. The ripple-like variations in the total power indicate that there are slight reflections at the boundaries of the FEA regions. The power radiated by the $A_{0}$ and $S_{0}$ modes are also depicted in the same figure. It is evident that the $A_{0}$ mode carries nearly $90 \%$ of the total radiated power, whereas the $S_{0}$ mode is becomes a more important source of loss at higher frequencies. The contrast in the ripple-like structure riding on top of the Lamb wave mode curves suggests that the absorbing boundary conditions in the FEA is more satisfactory for the $A_{0}$ mode. These results enable us to derive an equivalent circuit model of the membrane with physically based loss terms as described below.

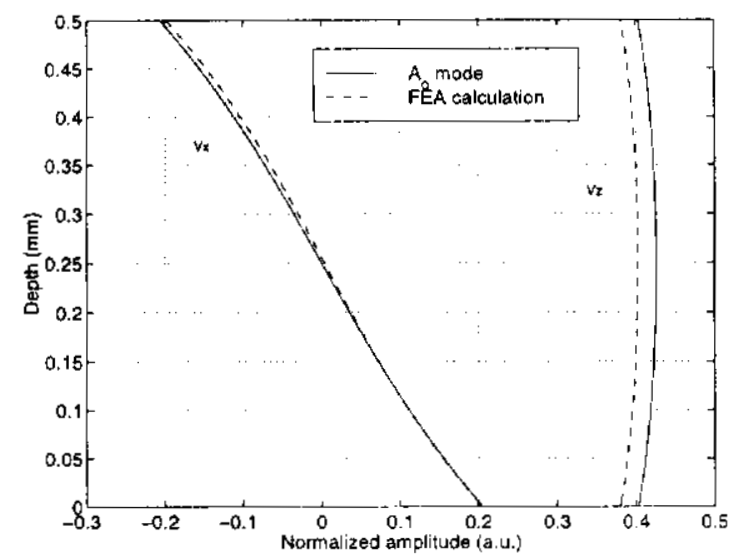

Fig. 4. Particle velocity field distribution of the $A_{0}$ mode and the FEA calculation at $1 \mathrm{MHz}\left(r_{1}=39.6 \mathrm{~mm}\right)$.

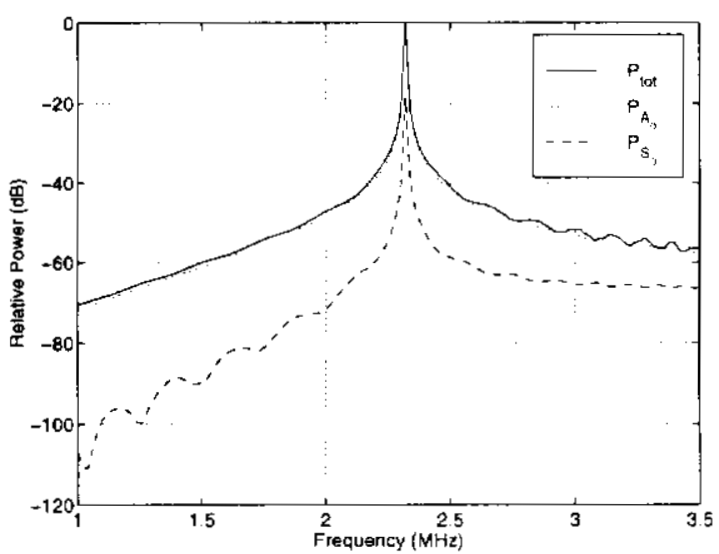

Fig. 5. Total radiated power calculated by Eq. 4 and the power levels of the $A_{0}$ and $S_{0}$ modes.

\section{Equivalent Circuit Model}

The ideal lossless membrane is modeled as a capacitor and an inductor in series representing its compliance and mass, respectively. Substrate loss can be included to the model as a series frequency dependent resistor. The value of this resistor can be found by 
finding the acoustic impedance $Z_{a}$ of the membrane from FEM results: If the applied pressure on element faces is $P$, the the total force on the membrane surface is $F_{\text {tot }}=\pi a^{2} P$. The average velocity of the membrane; $V_{\text {avg }}$ is given by

$$
V_{\text {avg }}=j \omega \frac{1}{\pi a^{2}} \int_{0}^{a} 2 \pi r u(r) d r
$$

where $u(r)$ is the membrane displacement found from the FEM analysis. The acoustic impedance of the membrane is, then, given by $Z_{a}=F_{\text {tot }} / V_{\text {avg }}$. As we claim that the real part of $Z_{a}$ accounts for the substrate loss, the resistance found by computing the power propagating out from the membrane, should be identical to $\operatorname{Re}\left(Z_{a}\right)$. The loss resistance is then given by $0.5 P_{\text {tot }} /\left|V_{\text {avg }}\right|^{2}$, where $P_{\text {tot }}$ is calculated using Eq. 4 .

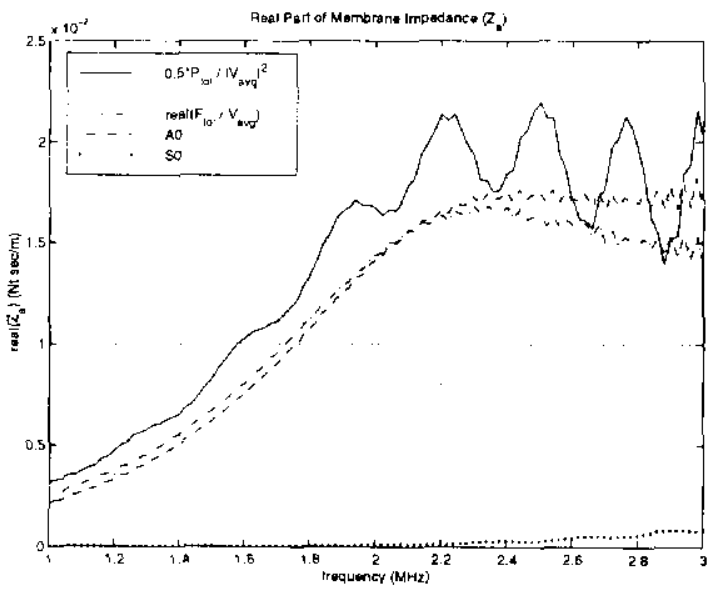

Fig. 6. Loss resistance of cMUT.

Figure 6 shows that the real part of the membrane impedances computed using the two suggested methods are indeed in agreement. The value of loss resistance is about $1 / 100$ of the impedance of air as the loading medium, hence, power loss will be $-20 \mathrm{~dB}$ with respect to the radiated power of an air transducer. The loss resistance can be further divided into terms each representing the loss due to a different propagating mode in the silicon substrate giving more insight into the physical loss mechanism. The result of this calculation for the $A_{0}$ and $S_{0}$ modes are also depicted in Fig. 6.

\section{Conclusion}

The main loss mechanism of cMUT is the radiation of energy to the silicon wafer. This energy is coupled to the propagating modes of the silicon wafer at the membrane-substrate junction. Finite element analysis results combined with the normal mode theory is used to show that the $A_{0}$ mode is the dominant propagation mode at low frequencies and to derive an equivalent circuit model for a lossy transducer membrane. This analysis enables us to adjust the membrane parameters to reduce the cross-coupling and loss due to these spurious wave modes of the system.

\section{REFERENCES}

[1] M. I. Haller and B. T. Khuri-Yakub, "A surface micromachined electrostatic ultrasonic air transduces," IEEE Trans. on Ultrasonics, Ferroelectrics and Frequency Control, vol. 43, no. 1, pp. 1-6, January 1996.

[2] P. Eccardt, K. Njederer, T. Scheiter, and C. Hierold, "Surface micromachined ultrasound transducers in CMOS technology," in Ultrasonics Symposium, San Antonio, Texas, November 1996, IEEE Cltrasonics, Ferroelectrics, and Frequency Control Society, pp. 959-962

[3] I. Ladabaum, X. C. Jin, H. T. Soh, A. Atalar, and B. T. Khuri-Yakub, "Surface micromachined capacitive ultrasonic transducers," IEEE Trans. on Ultrasonics, Ferroelectrics and Frequency Control, vol. 45, no. 3, pp. 678-690, May 1998.

[4] A. R. Levander, "Use of telegraphy equation to improve absorbing boundary efficiency for fourthorder acoustic wave finite difference schemes," Bull. Seismil. Soc. Am., , no. 75, pp. 1847-1852, June 1985.

[5] C. Cerjan, D. Kosloff, R. Kosloff, and M. Reshef, "A nonreflecting boundary condition for discrete acoustic and elastic wave equations," Geophysics, , no. 50, pp. 705-708, April 1985

[6] J. P. Berenger, "A perfectly matched layer for the absorption of electromagnetic waves," $J$. Comput. Phys., , no. 114 , pp. 185-200, 1994

[7] W. C. Chew and Q. H. Liu, "Lsing perfectly matched layers for elastodynamics," in IEEE Antennas Propagation Society International Symposium. IEEE Antennas Propagation Society, 1996, pp. 366-369.

[8] F. D. Hastings, J. B. Schneider, and S. L. Broschat, "Application of the perfectly matched layer (PML) absorbing boundary condition to elastic wave propagation," $J$. Acoust. Soc. Am., no. 100, pp. 3061-3069, November 1996.

[9] Q. Liu and J. Ta0, "The perfectly matched layer for acoustic waves in absorbing media," J. Acoust. Soc. Am., no. 102, pp. 2072-2082, October 1997.

[10] X. Yuan, D. Borup, J. W. Wiskin, M. Berggren, R. Eidens, and S. Johnson, "Formulation and validation of berenger's PML absorbing boundary for the FDTF simulation of acoustic scattering," IEEE Trans, on Ultrasonics, Ferroelectrics and Frequency Control, , no. 44, pp. 816-822, July 1997.

[11] G. F. Miller and H. Pursey, "The field and radiation impedance of mechanical radiators on the free surface of a semi-infinite isotropic solid," Proc. Royal Academy, pp. $521-541,1953$.

[12] B. A. Auld, Acoustic Fields and Waves in Solids, Krieger Publishing Co., 1990. 